\title{
Silencing Ku80 using small interfering RNA enhanced radiation sensitivity in vitro and in vivo
}

\author{
YOSHINORI NIMURA ${ }^{1,6}$, TETSUYA KAWATA ${ }^{2}$, KATSUHIRO UZAWA $^{3}$, JUNKO OKAMURA ${ }^{2}$, \\ CUIHUA LIU ${ }^{2}$, MASAYOSHI SAITO ${ }^{2}$, HIDEAKI SHIMADA ${ }^{1,4}$, NAOHIKO SEKI ${ }^{1,5}$, \\ AKIRA NAKAGAWARA ${ }^{6}$, HISAO ITO ${ }^{2}$, TAKENORI OCHIAI ${ }^{1,4}$ and HIDEKI TANZAWA ${ }^{1,3}$ \\ ${ }^{1} 21$ st Century Center of Excellence Program, ${ }^{2}$ Department of Radiology, ${ }^{3}$ Clinical Molecular Biology, \\ ${ }^{4}$ Academic Surgery, and ${ }^{5}$ Functional Genomics, Chiba University Graduate School of Medicine; \\ ${ }^{6}$ Division of Biochemistry, Chiba Cancer Center Research Institute, Chiba, Japan
}

Received February 5, 2007; Accepted March 21, 2007

\begin{abstract}
Ku80 is an important component of DNA doublestrand break repair, and Ku80 deficiency leads to extreme sensitivity to ionizing radiation. We studied whether radiation therapy combined with Ku80 silencing by small interfering RNA enhances radiation sensitivity in vitro and in vivo. Seven human cancer cell lines were transfected with Ku80 siRNA included in hemagglutinating virus of Japan envelope vector. H1299 cells were implanted into male BALB/C nu/nu nude mice treated with Ku80 siRNA and irradiation. The survival rate of cell lines transfected with Ku80 siRNA decreased by $10 \%$ to $26 \%$ with $2-$ Gy irradiation compared with untransfected cell lines. The gamma-H2AX phosphorylation-positive rates of Ku80 siRNA combined treatment $0.5 \mathrm{~h}$ after irradiation in A549 cells and $6 \mathrm{~h}$ in H1299 cells were significantly higher $(77.6 \%, \mathrm{p}=0.033$ and $76.7 \%, \mathrm{p}=0.026$, respectively), compared with the groups not treated with siRNA. H1299 xenograft tumors treated with combined therapy decreased in volume and re-grew slowly compared with radiation alone. Our results indicate that combined therapy consisting of Ku80 siRNA and irradiation contributes to inhibition of tumor growth and may be a novel strategy for cancer treatment.
\end{abstract}

Correspondence to: Professor Hideki Tanzawa, 21st Century Center of Excellence Program, Chiba University Graduate School of Medicine, 1-8-1 Inohana, Chuo-ku, Chiba 260-8677, Japan E-mail: tanzawap@faculty.chiba-u.jp

Abbreviations: DSBs, double-strand breaks; NHEJ, non-homologous end-joining; DNA-PKcs, DNA-dependent protease kinase catalytic subunits; siRNA, small interfering RNA; HVJ, hemagglutinating virus of Japan; HVJ-E, HVJ envelope; PBS, phosphate-buffered saline; xrs-5, X-ray-sensitive mutant 5; CHO K1, Chinese hamster ovary

Key words: Ku80, small interfering RNA, HVJ envelope, radiation sensitivity

\section{Introduction}

Radiation therapy is a standard treatment for patients with many kinds of cancers. Therapeutic strategies and protocols have been developed and the effectiveness of radiation therapy has greatly improved. However, it is difficult to predict the efficacy and side effects before therapy. Even in patients with the same stage of carcinoma, different responses and resistance to radiation therapy frequently affect the therapy. Radiation therapy with enhanced efficacy using a molecular technique is not yet established. A novel strategy based on a biologic mechanism should optimize the treatment of patients with carcinoma.

DNA double-strand breaks (DSBs) are potentially lethal DNA lesions induced by ionizing radiation $(1,2)$. DSBs can be repaired by homologous recombination or non-homologous end-joining (NHEJ) $(1,2)$. In mammals, NHEJ is especially important for repairing radiation-induced DSBs. Several factors, including $\mathrm{Ku} 70$, Ku80, DNA-dependent protease kinase catalytic subunits (DNA-PKcs), artemis, X-raycomplementation group 4, and DNA ligase IV, participate in this pathway (3-5). Ku works in a comparatively early stage. The Ku70/80 complex binds to the DSB ends, recruits DNA$\mathrm{PKcs}$, and initiates repair $(3,6)$. Ku deficiency leads to extreme sensitivity to radiation $(7,8)$.

Small interfering RNA (siRNA), has been used widely to silence gene expression, and has been evaluated as an attractive tool for use in therapeutics of many cancers (9-14). Some studies have reported that silencing the various repair genes, ATM, ATR, and DNA-PKcs (15,16), Rad51 (17), NBS1 $(18,19)$, and Mrel1 (20), increased radiation sensitivity. However, the efficacy of Ku80 silencing for radiation sensitivity has not yet been evaluated.

siRNA technology is a powerful method of gene downregulation; however, in vivo, it has been difficult to achieve high efficacy using the siRNA delivery system. The hemagglutinating virus of Japan (HVJ; Sendai virus) envelope vector is a new reagent for the transfection of DNA, protein, and oligonucleotides (21-26). The HVJ envelope (HVJ-E) vector was constructed with inactivated particles and therefore has no viral activity. Using the HVJ-E system, the efficiency of 
gene transfer into various cell lines is greatly enhanced, even when injected into organs directly in in vivo experiments (21-23).

We hypothesized that radiation therapy combined with Ku80 silencing might enhance radiation sensitivity, because of the reduced reparative ability of DSBs. In the current study, the expression of Ku80 was alternately inhibited using siRNA included in HVJ-E, and we studied in vitro and in vivo whether radiation sensitivity could be enhanced regardless of the type and radiation survival rate of the cancer cell lines.

\section{Materials and methods}

Cell lines and treatment. A549 and H1299 cells, which are lung carcinomas, were obtained from the American Type Culture Collection (Rockville, MD). A549 cells express normal p53, and H1299 cells are null for the p53 gene. TE13 (esophageal carcinoma), PANC-1 (pancreas carcinoma), MIAPaCa-2 (pancreas carcinoma), DU-145 (prostate carcinoma), and ME-180 (cervical carcinoma) cells were obtained from the Cell Resource Center for Biomedical Research Institute of Development, Aging and Cancer Tohoku University (Miyagi, Japan). All cells were maintained as a monolayer in Dulbecco's modified Eagle's medium (Sigma, St. Louis, MO), supplemented with $10 \%$ fetal bovine serum, and grown at $37^{\circ} \mathrm{C}$ in a humidified atmosphere of $5 \%$ carbon dioxide. Exponentially grown cells were used for all experiments.

Clonogenic assay. To evaluate radiation sensitivity, surviving fractions were measured using a standard colony-formation assay (27). Briefly, the cells were counted and the appropriate number seeded into 60-mm dishes in triplicate for each dose. Six hours later, when all cells had attached but not yet divided, the cells were irradiated with doses ranging from 0 to 6-Gy using an MBR-1520R-3 (Hitachi, Tokyo, Japan) generator operated under $150 \mathrm{kVp}$ and $20 \mathrm{~mA}$ with a $1-\mathrm{mm}$ aluminum filter. The dose rate was about $2-\mathrm{Gy} / \mathrm{minute}$. Colonies obtained after 10 to 14 days were stained with crystal violet and contained $>50$ cells.

siRNA transfection. The siRNA used for Ku80 gene silencing was designed by Qiagen (Valencia, CA). The target sequence was AAG CGA GTA ACC AGC TCA TAA, and the siRNA sense sequence was $\mathrm{r}(\mathrm{GCG}$ AGU AAC CAG CUC AUA A)dTdT.

Transfection of Ku80 siRNA was conducted with HVJ-E according to the manufacturer's recommendations (Ishihara Sangyo Kaisha, Ltd., Osaka, Japan). Briefly, cells were seeded into 6-well plates for $24 \mathrm{~h}$ before siRNA transfection. One microgram of siRNA was prepared to make complexes with HVJ-E according to the manufacturer's instructions (Ishihara Sangyo Kaisha, Ltd.). Several cell lines were transfected with Ku80 siRNA included in HVJ-E in medium supplemented with $10 \%$ fetal bovine serum. Forty hours after transfection, the cells were trypsinized and some were irradiated for clonogenic assay. The rest of the cells were lysed to extract protein for Western blotting.

Western blot analysis. Cells were lysed in lysis buffer [50 mM Tris-HCl (pH 8.0), $450 \mathrm{mM} \mathrm{NaCl}, 1 \%$ Triton X-100, $1 \mathrm{mM}$
EDTA (pH 8.0), and 0.6 mM PMSF] containing a protein inhibitor cocktail (4-benzenesulfonyl, fluoride, pepstatin A, E-64, bestatin, leupeptin, and aprotinin) (Sigma). Protein concentrations of lysates were determined using a protein assay kit (Bio-Rad, Hercules, CA). Forty micrograms of protein was loaded and electrophoresed on $10 \%$ sodium dodecyl sulfate polyacrylamide gels. After blotting the nitrocellulose membranes, the protein was probed with primary mouse monoclonal Ku70, Ku80 (Santa Cruz Biotechnology, Inc., Santa Cruz, CA), and B-actin (Sigma) antibodies then with secondary horseradish peroxidase-conjugated goat anti-mouse IgG antibody (Santa Cruz Biotechnology, Inc.). The immunoreactive bands were visualized using enhanced chemiluminescence on X-ray film (ECL Western Blotting Detection Reagent, GE Healthcare Bio-Sciences Corp., Piscataway, NJ).

Immunofluorescent staining for gamma-H2AX. Cells were seeded onto chamber slides (Nalge Nunc International, Naperville, IL) and transfected with the HVJ-E vector containing Ku80 siRNA for $40 \mathrm{~h}$ according to the manufacturer's instructions (Ishihara Sangyo Kaisha, Ltd.). After transfection, the cells were irradiated with 2-Gy or treated immediately without irradiation. Irradiated cells were treated using the following procedure after $30 \mathrm{~min}$ or $6 \mathrm{~h}$. The cells were fixed with $1 \%$ paraformaldehyde solution in phosphate-buffered saline (PBS) for $30 \mathrm{~min}$ at room temperature, soaked in $-20^{\circ} \mathrm{C}$ $99 \%$ methanol for $5 \mathrm{~min}$, and incubated in $3 \%$ bovine serum albumin with $0.2 \%$ Tween-20 in PBS for $1 \mathrm{~h}$ at $37^{\circ} \mathrm{C}$. The cells were probed in primary mouse monoclonal anti-phosphoH2A.X (ser139) antibody (Upstate Biotechnology, Lake Placid, NY) at a dilution of $1: 300$ for $1.5 \mathrm{~h}$ at $37^{\circ} \mathrm{C}$ and then in secondary Cy2-goat anti-rabbit $\mathrm{IgG}$ antibody at a dilution of 1:400 for $1 \mathrm{~h}$ at room temperature.

Fluorescence images were captured by Axioskop2 plus confocal microscopy equipped with a CCD camera and quantitated using AxioVisio Release 4.3 software (Carl Zeiss, Oberkochen, Germany). Significant colocalization of nuclear foci was determined by visualization of green spots on a DAPI background.

Gamma-H2AX foci were determined in five fields and at least 50 cells of each experiment. The cells were classified as positive when more than 10 foci were counted in the nucleus.

In vivo xenograft model. In vivo studies were carried out in accordance with the Guidelines for Animal Experimentation of Chiba University. Four-week-old male BALB/C nu/nu nude mice were obtained from Charles River Japan Inc. (Yokohama, Japan). To generate tumor xenografts, 5.0×106 viable H1299 cells were subcutaneously injected into the right hind legs. Tumors were measured in two dimensions with calipers and the volume was estimated using the following calculation: (major axis) $\mathrm{x}$ (minor axis) $\mathrm{x}$ (minor axis) $\mathrm{x} 1 / 2$.

We generated two protocols, one for one fraction and the other for five fractions. For one fraction, when the tumors reached $150-200 \mathrm{~mm}^{3}$ (day 0 ), the mice were randomly divided into three groups: control (no radiation; $n=11$ ), radiation alone (4-Gy in one fraction on day $1 ; n=6$ ), and Ku80 siRNA treatment with radiation (siRNA injection on day 0 and $4-G y$ in one fraction on day $1 ; n=6$ ). For five 
fractions, when the tumors reached $750-1000 \mathrm{~mm}^{3}$ (day 0 ), the mice were randomly divided into three groups: control (no radiation; $\mathrm{n}=10$ ), radiation alone (4-Gy in one fraction on days $1,2,3,4$, and $5 ; n=7)$, and Ku80 siRNA treatment with radiation (siRNA injection on days 2 and 4, and 4-Gy in one fraction on days $1,2,3,4$, and $5 ; n=8)$.

Gene delivery. H1299 xenograft tumors in the hind legs of nude mice were treated with Ku80 siRNA included in HVJ-E. The HVJ-E complex included Ku80 siRNA prepared as previously described. Ku80 siRNA was injected directly into the tumors. Each injection was diluted in a total volume of $50 \mu \mathrm{l}$ of $0.9 \%$ sodium chloride solution and administered in one pass using a 27 -gauge needle.

Statistical analysis. Statistical analysis was performed using SigmaStat 3.1 (Point Richmond, CA). For a single comparison, the level of statistical significance was confirmed using Student's t test. $\mathrm{p}<0.05$ were considered statistically significant.

\section{Results}

Down-regulation of Ku80 protein expression by siRNA in A549 and H1299 cells. To determine whether Ku80 silencing contributes to radiation sensitivity, two siRNAs were designed and screened for the ability to down-regulate target protein expression. To determine the best protocol for silencing $\mathrm{Ku} 80$ protein expression, the cells were transfected with siRNA/HVJ-E complexes at various times, and protein extracts were obtained from 6 to $96 \mathrm{~h}$ after transfection. Western blot analyses showed that siRNA/HVJ-E complexes minimized $\mathrm{Ku} 80$ expression $40 \mathrm{~h}$ after transfection compared with untransfected cells (data not shown). We used $40 \mathrm{~h}$ of treatment with siRNA for the experiments.

Western blot analysis of Ku80 protein expression in A549 and H1299 cells was performed $40 \mathrm{~h}$ after transfection with Ku80 siRNA (Fig. 1A). Ku80 protein expression was suppressed, indicating successful gene silencing by siRNA. In cells transfected with Ku80 siRNA, Ku80 protein decreased compared with the transfected HVJ-E or the untransfected cells. Interestingly, although the cells were transfected with $\mathrm{Ku} 80$ siRNA, Ku70 protein expression also was suppressed and correlated with Ku80 expression level in both cell lines. The A549 cells are wild-type cells of the p53 gene and the H1299 cells are the null type. The efficiency of the suppression did not depend on the $p 53$ gene status.

Cellular sensitivity after treatment with Ku80 siRNA. The A549 and H1299 cells exhibited a typical clonogenic survival curve with a shoulder signifying cellular repair capacity. Doses of 2, 4, and 6-Gy of irradiation alone killed about 31, 60 , and $84 \%$ of the A549 cells, and 21, 53, and $76 \%$ of the H1299 cells, respectively (Fig. 1B). Furthermore, the survival rates of cells transfected with HVJ-E alone were close to the rate for the original cells. However, Ku80 siRNA enhanced cell killing in 57,86, and 96\% of the A549 cells and 35, 71, and $90 \%$ of the H1299 cells, respectively. siRNA inhibition of Ku80 protein confirmed enhanced radiation sensitivity in siRNA-transfected cells compared with the transfected
A

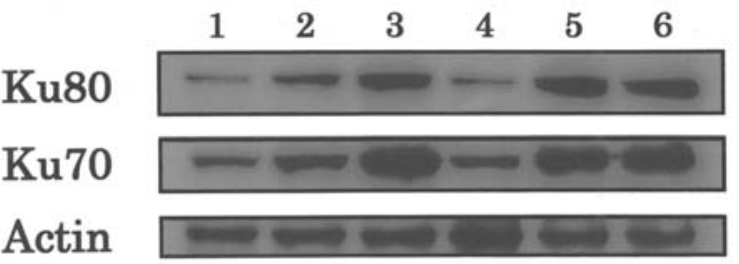

B
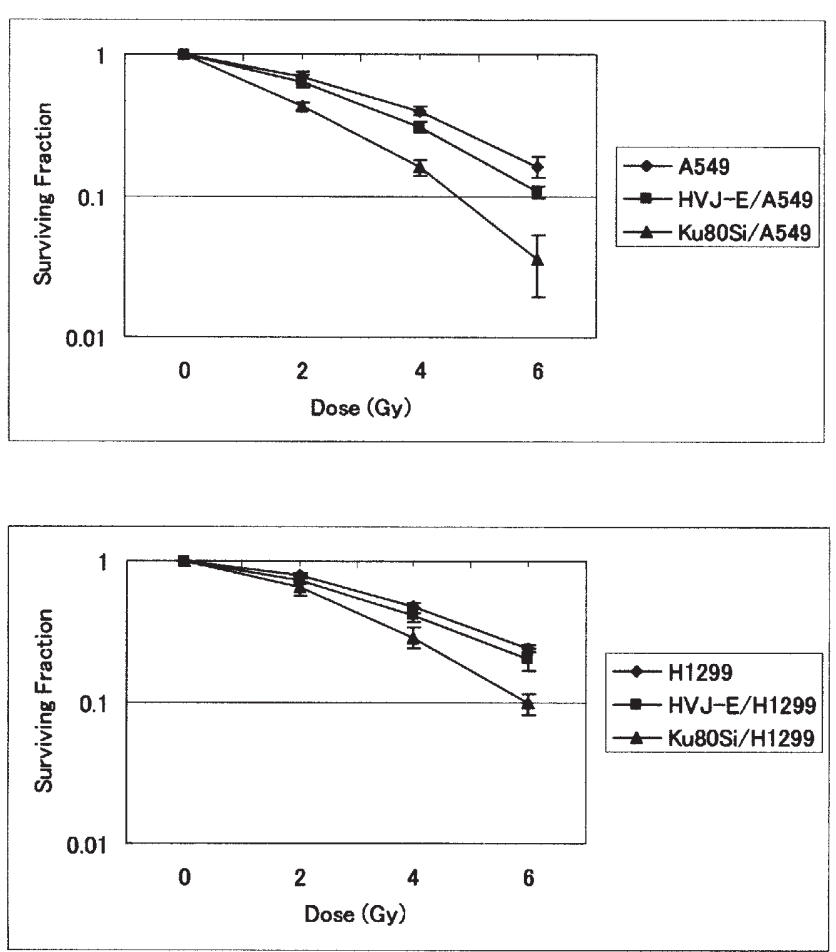

Figure 1. Down-regulation of Ku80 protein and radiation sensitivity in A549 and H1299 cells. Western blotting: after treatment with HVJ-E including $\mathrm{Ku} 80$ siRNA for $40 \mathrm{~h}$, the protein expression of Ku 80 is suppressed compared with the cell lines without siRNA or those treated with only HVJ-E. Actin serves as a loading control. Lane 1, Ku80si/A549; lane 2, HVJ-E/A549; lane 3, A549; lane 4, Ku80si/H1299; lane 5, HVJ-E/H1299; and lane 6, H1299 (A). Clonogenic assay: in the cell line treated with Ku80 siRNA, the surviving fraction of the A549 cells treated with 2-Gy irradiation is reduced by $26 \%$, and the surviving fraction of the H1299 cells is reduced by $14 \%$ compared with the untreated cell line. Radiation sensitivity is enhanced by treatment with Ku80 siRNA. All experiments were performed in triplicate. Data are presented as the mean $\pm \mathrm{SE}(\mathrm{B})$.

HVJ-E or the untransfected cells. We focused on the results of 2-Gy irradiation, which is the usual dose for a single fraction in the clinic. The increased radiation sensitivity at 2-Gy irradiation corresponded to 26 and $14 \%$ for $\mathrm{Ku} 80$ silencing in the A549 and H1299 cells, respectively.

Radiation sensitivity enhancement by Ku80 siRNA in several human cancer cell lines. After controlling and suppressing Ku80 in tumor cells alternately using siRNA, it was important to verify if radiation sensitivity can be enhanced regardless of the modality and radiation survival rate of a tumor. Another five cancer cell lines, TE13, PCNA-1, MIAPaCa-2, DU-145, and ME-180, were selected for the Ku80 siRNA experiments, because radiation therapy is usually effective for these cancers. Western blot analyses of Ku80 protein expression in all cell 
A

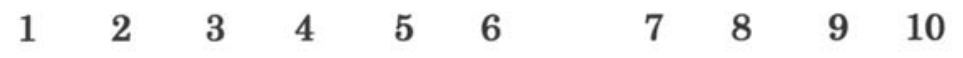

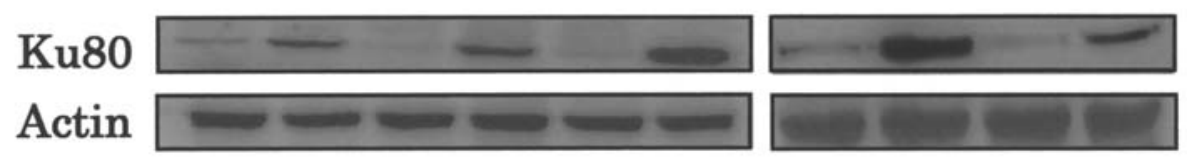

B
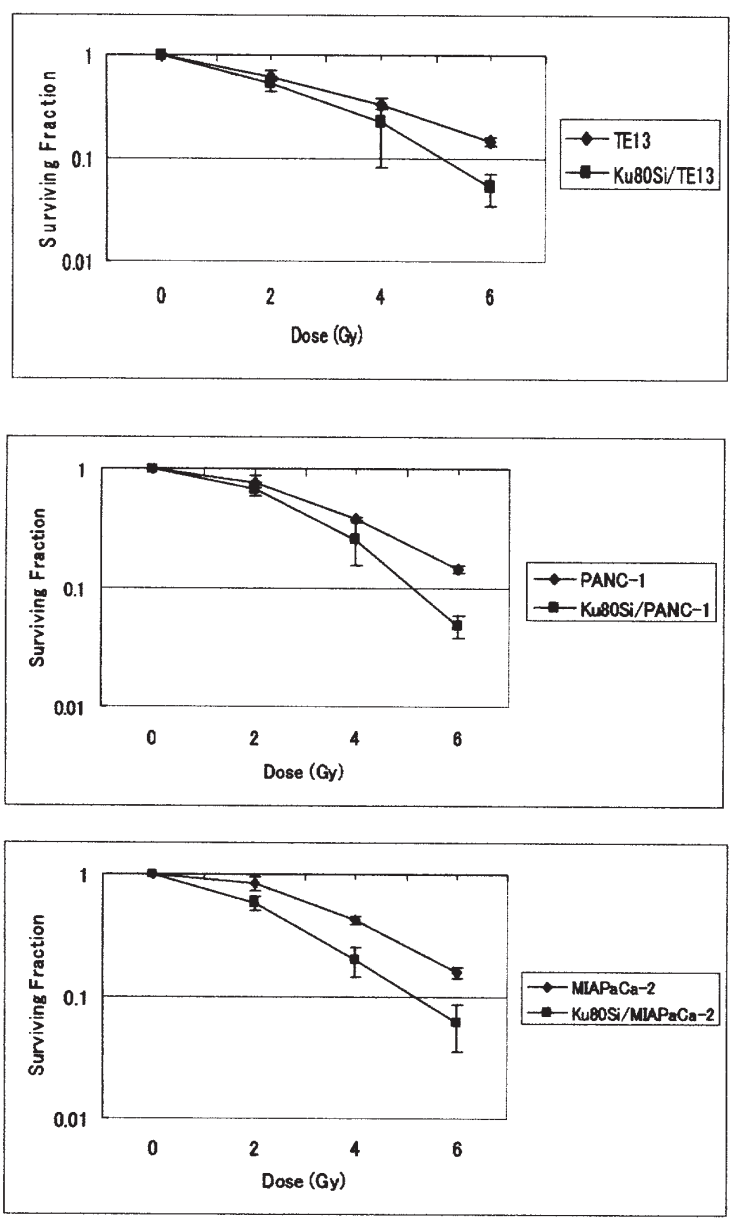

lines were performed as described previously. After transfection of Ku80 siRNA, Ku80 protein expression was suppressed compared with the untransfected cells (Fig. 2A).

In the cell lines treated with siRNA, surviving fractions of TE13 treated with 2-Gy irradiation decreased by $10 \%$ that of PANC- 1 by $11 \%$, that of MIAPaCa- 2 by $25 \%$, that of DU145 by $15 \%$, and that of ME- 180 by $16 \%$ compared with untreated cell lines (Fig. 2B). We observed enhanced radiation sensitivity regardless of the cancer type.

Reduced reparative ability of DSBs resulting from combined radiation and siRNA treatment. Ku80 siRNA apparently enhanced radiation sensitivity in several human cancer cell lines. According to the biologic mechanism, we hypothesized that radiation therapy combined with $\mathrm{Ku} 80$ silencing would reduce the DSBs reparative ability. Thus, immunofluorescence detection of gamma-H2AX nuclear foci was performed to detect DSBs.

Gamma-H2AX foci were detected after 2-Gy irradiation in the A549 and H1299 cells. Gamma-H2AX foci stained
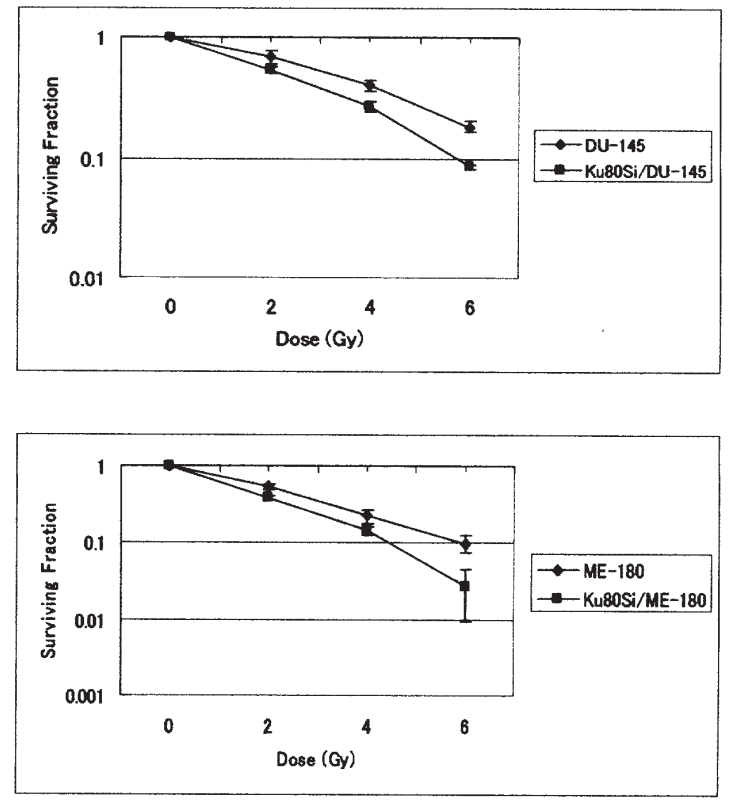

Figure 2. Enhanced radiation sensitivity by Ku80 siRNA in several human cancer cell lines. Western blotting: Ku80 expression of all cell lines treated with siRNA is suppressed compared with the cell line without transfection. Actin serves as a loading control. Lane 1, Ku80si/TE13; lane 2, TE13; lane 3, Ku80si/PANC-1; lane 4, PANC-1; lane 5, Ku80si/MIAPaCa-2; lane 6, MIAPaCa-2; lane 7, Ku80si/DU-145; lane 8, DU-145; lane 9, Ku80si/ME-180; and lane 10, ME-180 (A). Clonogenic assay: in the cell lines treated with siRNA, the fraction of the TE13 cells surviving 2-Gy irradiation is decreased by $10 \%$, that of PANC- 1 by $11 \%$, that of MIAPaCa- 2 by $25 \%$, that of DU-145 by $15 \%$, and that of ME- 180 by $16 \%$ compared with the untreated cell line. All experiments were performed in triplicate. Data are presented as the mean $\pm \mathrm{SE}(\mathrm{B})$.

green; all cells counterstained blue with DAPI for nuclear DNA (data not shown). When $>10$ gamma-H2AX foci were seen in the cell, it was determined as positive. In both cell lines, positive rates of gamma-H2AX foci immediately increased and decreased over time. The two positive rates with siRNA, at $0.5 \mathrm{~h}$ after irradiation in A549 cells and at $6 \mathrm{~h}$ in $\mathrm{H} 1299$ cells, were significantly higher by $77.6 \%$ (95\% CI, $70-85 ; \mathrm{p}=0.033)$ and $76.7 \%$ (95\% CI, 68-86; $\mathrm{p}=0.026)$ compared with no siRNA treatment, 64.1 and $57.5 \%$, respectively (Fig. 3). This suggested that the ability of the DSB repair decreased after radiation and siRNA treatment compared with radiation alone.

Suppression of tumor growth by Ku80 siRNA in H1299 xenograft models. To examine further effects of Ku80 siRNA, we conducted in vivo experiments with H1299 xenograft tumors. We used the in vivo radiation dose of 4-Gy to simplify ascertaining the effect after treatment.

Before testing the two protocols, we first investigated the post-irradiation effects to estimate the efficiency of Ku80 
A

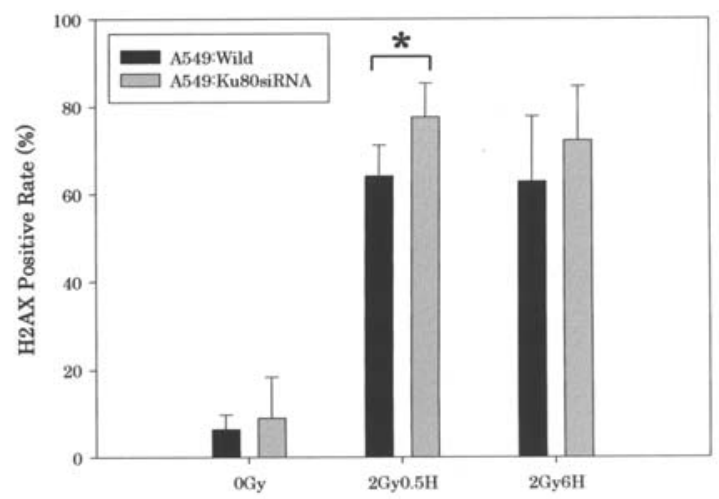

B

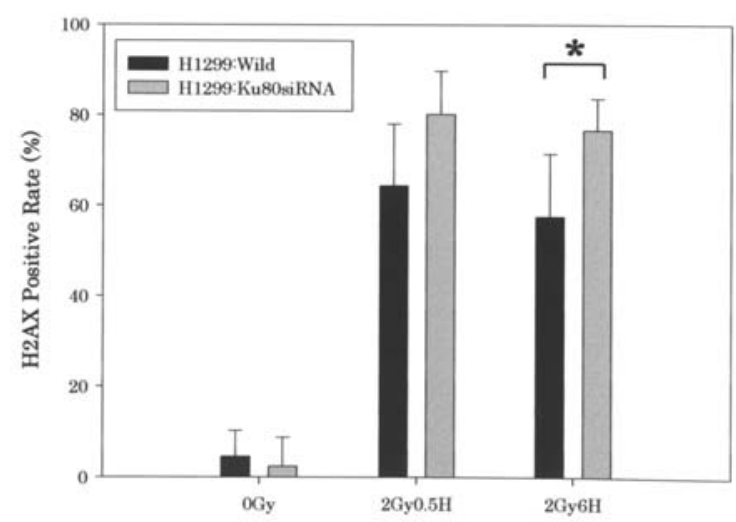

Figure 3. Positive rate of gamma-H2AX foci of A549 and H1299 cells stained 0.5 and $6 \mathrm{~h}$ after 2-Gy irradiation. In the A549 cells, the positive rate with siRNA treatment $0.5 \mathrm{~h}$ after irradiation is significantly higher than that with no siRNA treatment ( $\mathrm{p}=0.033$ ) (A). In the H1299 cells, the positive rate with siRNA treatment $6 \mathrm{~h}$ after irradiation is significantly higher than that with no siRNA treatment $(\mathrm{p}=0.026)$. Data are presented as the mean $\pm \mathrm{SE} . \mathrm{H}$, hours $(B)$.

siRNA. When tumor volume reached $150-200 \mathrm{~mm}^{3}$, the mice were randomly divided into four groups: control $(n=5)$, radiation alone $(n=4), H V J-E$ vector treatment with radiation $(\mathrm{n}=5)$, and Ku80 siRNA treatment with radiation $(\mathrm{n}=6)$. After 4-Gy radiation, most tumors decreased in volume within 6 days and then began to increase again (Fig. 4A). Fig. 4B shows the percentage of the minimum volumes of each group compared with day $1(100 \%)$. The rate of the reduction in volume of the tumors treated with Ku80 siRNA and radiation was the highest compared with the other groups. The scores of the remaining tumor were $72.95 \%$ for radiation alone, $72.70 \%$ for HVJ-E with radiation, and $56.02 \%$ for Ku80 siRNA with radiation. Even if one treatment was performed, an anti-tumor effect was expected from combination therapy with Ku80 siRNA and irradiation.

Delay in tumor growth by Ku80 siRNA for long-term observation. After confirming the suppression of tumor growth by Ku80 siRNA in vivo, the next goal was to follow for a long period the tumors treated with one fraction. When tumor volume reached $150-200 \mathrm{~mm}^{3}$, the mice were randomly divided into three groups: control $(n=11)$, radiation alone $(n=6)$, and Ku80 siRNA treatment with radiation $(n=6)$. The volume of the tumors in the two groups treated with radiation alone and combination therapy with Ku80 siRNA decreased
A

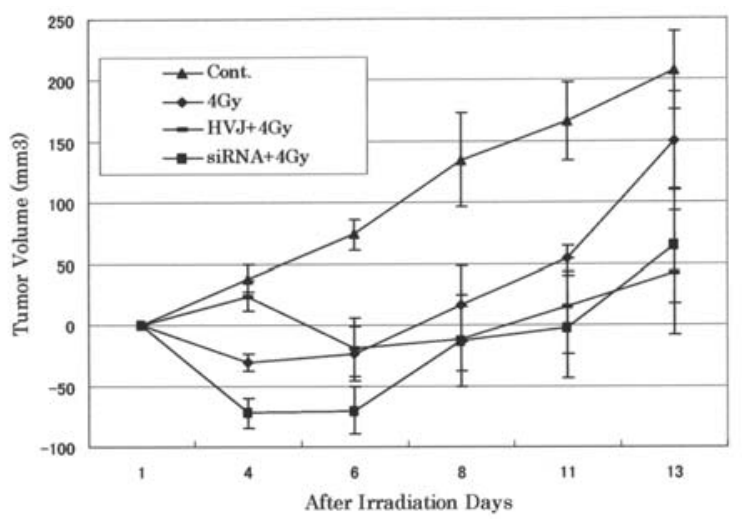

B

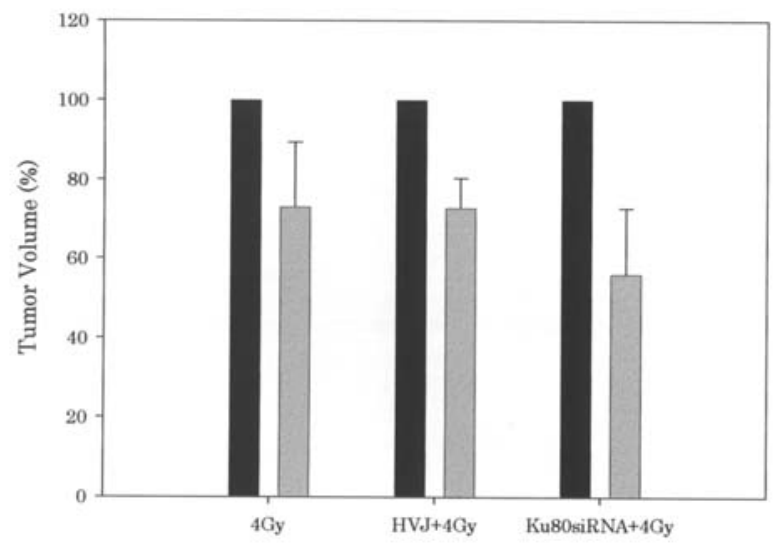

Figure 4. Enhanced tumor growth suppression by Ku80 siRNA combined with radiation therapy. Tumor growth curves for H1299 xenograft tumors treated with irradiation alone, HVJ-E vector, or Ku80 siRNA with irradiation. Quantification of $\mathrm{H} 1299$ xenograft increases $(>0)$ or decreases $(<0)$ tumor volumes compared with day 1 (A). The minimum volume of each tumor after irradiation was determined and calculated as a percentage in the various treatment groups compared with day $1(100 \%)$ when the tumor was irradiated. Tumor volumes were measured two or three times weekly. Data are presented as the mean $\pm \mathrm{SE}(\mathrm{B})$.

until day 6 and re-grew in almost parallel fashion (Fig. 5A). By day 32, the increased volume of tumors treated with radiation alone was $404.4 \pm 60.2 \mathrm{~mm}^{3}$ and that of combination therapy with $\mathrm{Ku} 80$ siRNA was $308.5 \pm 38.2 \mathrm{~mm}^{3}$, indicating that tumor treated with combination therapy decreased to $76 \%$ of the volume of those treated with radiation alone.

The next trial consisted of 5 days of irradiation. To irradiate with five fractions, we waited until the tumor volume reached $750-1000 \mathrm{~mm}^{3}$, at which point the mice were randomly divided into three groups: control $(n=10)$, radiation alone $(n=7)$, and Ku80 siRNA treatment with radiation $(n=8)$. Irradiation was administered from days 1-5, and Ku80 siRNA was injected into the tumors on days 2 and 4 . Until day 15, there was no difference in tumor growth in the two groups, which were treated with radiation alone and combined Ku80 siRNA. After day 15, combination therapy with Ku80 siRNA and radiation resulted in delayed tumor growth (Fig. 5B). By day 32 , the increased tumor volume after treatment with five fractions alone was $1476.4 \pm 412.5 \mathrm{~mm}^{3}$ and that of five fractions with two Ku80 siRNA treatments was $830.9 \pm 93.8 \mathrm{~mm}^{3}$, indicating that Ku80 siRNA led to a $56 \%$ reduction in volume compared with radiation alone. For example, the proliferation time for the H1299 cell tumor to increase to $200 \mathrm{~mm}^{3}$ was 
A

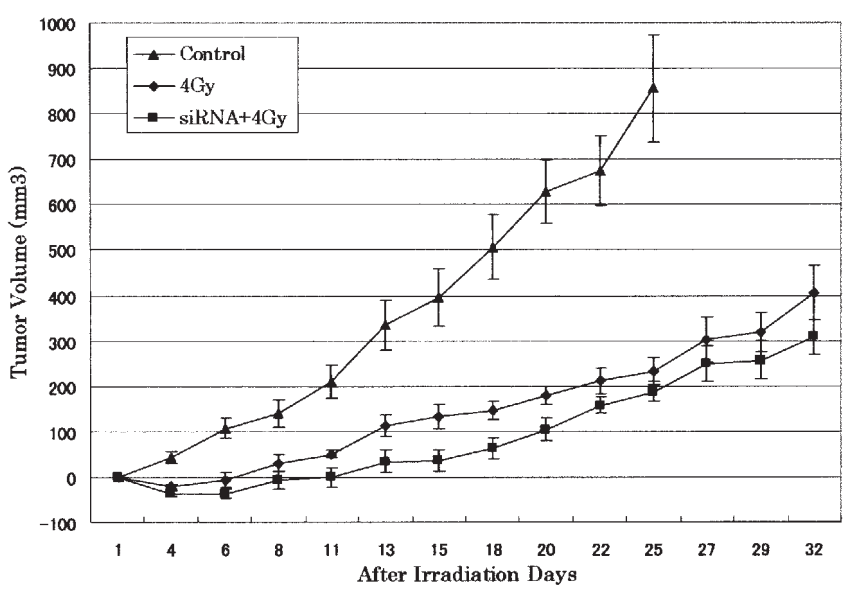

B

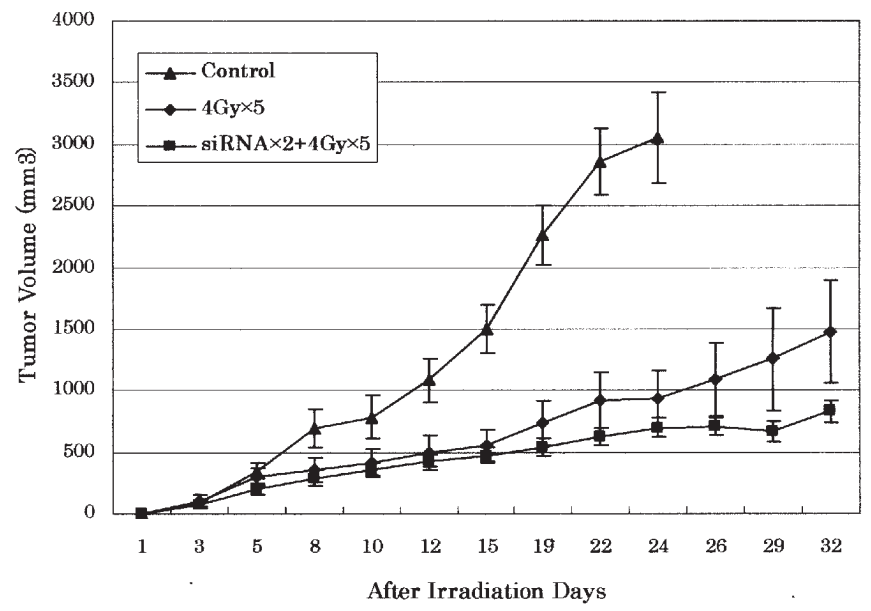

Figure 5. Growth delay curves of H1299 xenograft tumors treated with Ku80 siRNA and long-term irradiation. H1299 xenograft tumors were treated with one Ku80 siRNA treatment on day 0 and one fraction on day 1 (A). Tumors were treated with two Ku80 siRNA treatments on days 2 and 4 and five fractions from days 1 to 5 . Data are presented as the mean \pm SE (B)

about 22 days for the group treated with radiation alone. However, for the group treated with Ku80 siRNA and radiation, it took $>32$ days, which indicated a 10-day delay in tumor growth observed after using Ku80 siRNA.

\section{Discussion}

Ku80 plays an important role in repairing DNA DSBs, which are common in the DNA damage produced by irradiation (6). $\mathrm{Ku}$ was identified as an autoimmune antigen from the sera of a Japanese patient with scleroderma polymyosis overlap syndrome (28). An X-ray-sensitive mutant 5, xrs-5, was isolated from Chinese hamster ovary cell line (CHO K1) which was wild-type of Ku80 and extremely sensitive to irradiation (29). However, after transfection of Ku80 into xrs-5, the cells regained radiation resistance to the same level as CHO K1 (30). Therefore, we focused on Ku80 and hypothesized that silencing Ku80 might enhance radiation sensitivity and be advantageous in treating cancer with irradiation.
In this study, Ku80 protein was suppressed successfully by Ku80 siRNA in vitro. Although we chose Ku80 sequences, $\mathrm{Ku} 70$ protein expression also was suppressed after transfection with $\mathrm{Ku} 80$ siRNA. Loss of one $\mathrm{Ku}$ protein was reported to result in a significant decrease in the other $\mathrm{Ku}$ protein, because heterodimerization was required to stabilize each $\mathrm{Ku}$ protein (29). Furthermore, heterodimerization between Ku70 and $\mathrm{Ku} 80$ is essential for DSBs repair and also important in activation of DNA-PKs, which is a main function of Ku80 protein (3). Based on these facts, down-regulation of Ku80 protein by siRNA might have blocked heterodimerization with $\mathrm{Ku} 70$, thus inhibiting DNA repair after radiation.

We conducted an additional experiment to prove the inhibition of DNA DSBs repair after using Ku80 siRNA. Immunofluorescence detection of gamma-H2AX nuclear foci is used to detect DNA DSBs. The histone $\mathrm{H} 2 \mathrm{AX}$ protein is a variant member of the $\mathrm{H} 2 \mathrm{~A}$ family of histones that is rapidly phosphorylated at the ser139 residue in response to radiationinduced DNA DSBs, resulting in the formation of nuclear foci (31-33). The prolonged and multiple expressions of H2AX foci after irradiation suggests decreased repair of DNA DSBs (32). Thus, immunofluorescence detection of gamma-H2AX nuclear foci was performed to visualize radiation-induced DSBs after treatment with Ku80 siRNA and to investigate why the radiation sensitivity increased. As expected, combination therapy resulted in enhanced radiation sensitivity. This effect correlated with gamma-H2AX staining, suggesting that the enhanced radiation sensitivity resulted from suppression of the Ku80 protein, which inhibited the ability of the DNA DSBs to repair themselves.

siRNA is expected to be the new therapeutic agent for cancer treatment (9-14). Although various procedures, for example, intravenous and direct injection, are being considered, the important issue is how the anti-tumor effect is acquired efficiently. In the current study, we selected the HVJ-E vector, which has been reported to be a new tool for the transfection reagents and was evaluated as useful and safe in vitro and in vivo (21-23). We also focused on injecting siRNA included in the HVJ-E vector directly into the tumor in vivo. The HVJ-E vector has been extremely safe to handle and efficient for gene transfer, and it might be a powerful candidate for future clinical use.

When cells were transfected with Ku80 siRNA by HVJ-E, $\mathrm{Ku} 80$ protein suppression was observed between 18 and $96 \mathrm{~h}$ after transfection (data not shown). Thus, we selected $40 \mathrm{~h}$ in vitro and $20 \mathrm{~h}$ in vivo after transfection for initiating treatment with radiation therapy to optimize the efficacy. When we used five fractions in vivo, we injected siRNA on days 2 and 4, which was $20 \mathrm{~h}$ before the radiation on days 3 and day 5. Ito et al reported that Rad51 siRNA suppressed protein expression in HeLa cells between 1 and 4 days after transfection and enhanced sensitivity to cisplatin during that period (17). Although each siRNA and cells have their own ability and characteristics, siRNA usually prevents protein expression for a few days; thus, the best timing must be considered for the combination therapy based on efficacy.

In the current study, the cells were treated with one transfection of Ku80 siRNA and one session of irradiation in vitro. Even if one treatment was administered, combination therapy with Ku80 siRNA and irradiation obviously enhanced 
radiation sensitivity in several types of cancer cell lines. In vivo, H1299 xenograft tumors were treated twice with siRNA and five fractions, and the tumor volume decreased compared with radiation alone. However, most patients receive more than 20 fractions in normal clinical practice. So, in the future, we have to establish a regimen close to the clinical protocol, i.e., several treatments with Ku80 siRNA and irradiation.

Since not only malignant tumor cells but also normal tissues are exposed, there are many restrictions in the irradiation procedures, e.g., radiation times and tumor location. If the Ku80 expression in the tumor is inhibited by siRNA and the treatment that enhances radiation sensitivity can be performed for the patients, we may expect improvement in the efficiency of radiation therapy. Furthermore, the indication also may be extended to tumors that have not been targets of radiation therapy.

We showed that Ku80 siRNA included in the HVJ-E vector enhanced the radiation sensitivity of several human cancer cell lines regardless of the cancer type and radiation survival rate in vitro. Furthermore, radiation therapy combined with Ku80 siRNA resulted in inhibited tumor growth in vivo. Our results indicated that the combination of Ku80 siRNA and irradiation may be a useful novel strategy for cancer treatment considering the biological characteristics.

\section{Acknowledgments}

The authors are grateful to Drs Craig W. Stevens and Masaki Takiguchi for support. This study was financially supported by the 21st Century Center of Excellent program at Chiba University, and Grant-in-Aid for Scientific Research on Priority Areas (C) (grant numbers 16591189 and 17591250).

\section{References}

1. Haber JE: Partners and pathways repairing a double-strand break. Trends Genet 16: 259-264, 2000.

2. Hoeijmakers JH: Genome maintenance mechanisms for preventing cancer. Nature 411: 366-374, 2001.

3. Jin S and Weaver DT: Double-strand break repair by $\mathrm{Ku} 70$ requires heterodimerization with $\mathrm{Ku} 80$ and DNA binding functions. EMBO J 16: 6874-6885, 1997.

4. Nick McElhinny SA, Snowden CM, McCarville J and Ramsden DA: Ku recruits the XRCC4-ligase IV complex to DNA ends. Mol Cell Biol 20: 2996-3003, 2000.

5. Bassing $\mathrm{CH}$, Swat W and Alt FW: The mechanism and regulation of chromosomal V(D)J recombination. Cell 109: S45-S55, 2002.

6. Ramsden DA and Gellert M: Ku protein stimulates DNA end joining by mammalian DNA ligases: a direct role for $\mathrm{Ku}$ in repair of DNA double-strand breaks. EMBO J 17: 609-614, 1998.

7. Nussenzweig A, Chen C, da Costa Soares V, Sanchez M, Sokol K, Nussenzweig MC and Li GC: Requirement for Ku80 in growth and immunoglobulin $\mathrm{V}(\mathrm{D}) \mathrm{J}$ recombination. Nature 382: 551-555, 1996.

8. Zhu C, Bogue MA, Lim DS, Hasty P and Roth DB: Ku86deficient mice exhibit severe combined immunodeficiency and defective processing of $\mathrm{V}(\mathrm{D}) \mathrm{J}$ recombination intermediates. Cell 86: 379-389, 1996.

9. Zhang X, Chen ZG, Choe MS, Lin Y, Sun SY, Wieand HS, Shin HJ, Chen A, Khuri FR and Shin DM: Tumor growth inhibition by simultaneously blocking epidermal growth factor receptor and cyclooxygenase- 2 in a xenograft model. Clin Cancer Res 11: 6261-6269, 2005.

10. Gao L, Zhang L, Hu J, Li F, Shao Y, Zhao D, Kalvakolanu DV, Kopecko DJ, Zhao X and Xu DQ: Down-regulation of signal transducer and activator of transcription 3 expression using vector-based small interfering RNAs suppresses growth of human prostate tumor in vivo. Clin Cancer Res 11: 6333-6341, 2005.
11. Ito T, Hashimoto Y, Tanaka E, Kan T, Tsunoda S, Sato F, Higashiyama M, Okumura T and Shimada Y: An inducible short-hairpin RNA vector against osteopontin reduces metastatic potential of human esophageal squamous cell carcinoma in vitro and in vivo. Clin Cancer Res 12: 1308-1316, 2006.

12. Halder J, Kamat AA, Landen CN Jr, Han LY, Lutgendorf SK, Lin YG, Merritt WM, Jennings NB, Chavez-Reyes A, Coleman RL, Gershenson DM, Schmandt R, Cole SW, Lopez-Berestein G and Sood AK: Focal adhesion kinase targeting using in vivo short interfering RNA delivery in neutral liposomes for ovarian carcinoma therapy. Clin Cancer Res 12: 4916-4924, 2006

13. Amarzguioui M, Peng Q, Wiiger MT, Vasovic V, Babaie E, Holen T, Nesland JM and Prydz $\mathrm{H}$ : Ex vivo and in vivo delivery of anti-tissue factor short interfering RNA inhibits mouse pulmonary metastasis of B16 melanoma cells. Clin Cancer Res 12: 4055-4061, 2006

14. Hosaka S, Nakatsura T, Tsukamoto H, Hatayama T, Baba $H$ and Nishimura Y: Synthetic small interfering RNA targeting heat shock protein 105 induces apoptosis of various cancer cells both in vitro and in vivo. Cancer Sci 97: 623-632, 2006.

15. Peng Y, Zhang Q, Nagasawa H, Okayasu R, Liber HL and Bedford JS: Silencing expression of the catalytic subunit of DNA-dependent protein kinase by small interfering RNA sensitizes human cells for radiation-induced chromosome damage, cell killing, and mutation. Cancer Res 62: 6400-6404, 2002.

16. Collis SJ, Swartz MJ, Nelson WG and De Weese TL: Enhanced radiation and chemotherapy-mediated cell killing of human cancer cells by small inhibitory RNA silencing of DNA repair factors. Cancer Res 63: 1550-1554, 2003.

17. Ito M, Yamamoto S, Nimura K, Hiraoka K, Tamai $K$ and Kaneda Y: Rad51 siRNA delivered by HVJ envelope vector enhances the anti-cancer effect of cisplatin. J Gene Med 7: 1044-1052, 2005.

18. Zhang Y, Lim CUK, Williams ES, Zhou J, Zhang Q, Fox MH, Bailey SM and Liber HL: NBS1 knockdown by small interfering RNA increases ionizing radiation mutagenesis and telomere association in human cells. Cancer Res 65: 5544-5553, 2005.

19. Ohnishi K, Scuric Z, Schiestl RH, Okamoto N, Takahashi A and Ohnishi T: siRNA targeting NBS1 or XIAP increases radiation sensitivity of human cancer cells independent of TP53 satus. Radiat Res 166: 454-462, 2006.

20. Xu M, Myerson RJ, Hunt C, Kumar S, Moros EG, Straube WL and Roti JL: Transfection of human tumor cells with Mre11 SiRNA and the increase in radiation sensitivity and the reduction in heat-induced radiosensitization. Int $\mathbf{J}$ Hyperthermia 20 157-162, 2004.

21. Kaneda Y, Nakajima T, Nishikawa T, Yamamoto S, Ikegami H, Suzuki N, Nakamura H, Morishita R and Kotani H: Hemagglutinating virus of Japan (HVJ) envelope vector as a versatile gene delivery system. Mol Ther 6: 219-226, 2002.

22. Shimamura M, Morishita R, Endoh M, Oshima K, Aoki M, Waguri S, Uchiyama Y and Kaneda Y: HVJ-envelope vector for gene transfer into central nervous system. Biochem Biophys Res Commun 300: 464-471, 2003.

23. Nakamura H, Kimura T, Ikegami H, Ogita K, Koyama S, Shimoya K, Tsujie T, Koyama M, Kaneda Y and Murata Y: Highly efficient and minimally invasive in vivo gene transfer to the mouse uterus using haemagglutinating virus of Japan (HVJ) envelope vector. Mol Hum Reprod 9: 603-609, 2003.

24. Kojima C, Hashimoto A, Yabuta I, Hirose M, Hashimoto S, Kanaho Y, Sumimoto H, Ikegami T and Sabe H: Regulation of Bin1 SH3 domain binding by phosphoinositides. EMBO J 23: 4413-4422, 2004.

25. Ohnuma K, Yamochi T, Uchiyama M, Nishibashi K, Yoshikawa N, Shimizu N, Iwata S, Tanaka H, Dang NH and Morimoto C: CD26 up-regulates expression of CD86 on antigenpresenting cells by means of caveolin-1. Proc Natl Acad Sci USA 101: 14186-14191, 2004.

26. Sumi K, Yokozeki H, Wu MH, Satoh T, Kaneda Y, Takeda K, Akira $\mathrm{S}$ and Nishioka $\mathrm{K}$ : In vivo transfection of a cis element 'decoy' against signal transducers and activators of the transcription 6 (STAT6) binding site ameliorates the response of contact hypersensitivity. Gene Ther 11: 1763-1771, 2004.

27. Nimura Y, Ismail SM, Kurimas A, Chen DJ and Stevens CW: DNA-PK and ATM are required for radiation-enhanced integration. Radiat Res 157: 562-567, 2002. 
28. Mimori T, Akizuki M, Yamagata H, Inada S, Yoshida S and Homma M: Characterization of a high molecular weight acidic nuclear protein recognized by autoantibodies in sera from patients with polymyositis-scleroderma overlap. J Clin Invest 68: 611-620, 1981 .

29. Singleton BK, Priestley A, Steingrimsdottir H, Gell D, Blunt T, Jackson SP, Lehmann AR and Jeggo PA: Molecular and biochemical characterization of xrs mutants defective in Ku80. Mol Cell Biol 17: 1264-1273, 1997.

30. Stevens CW, Stamato TD, Mauldin SK, Getts RC, Zeng M and Cerniglia GJ: Radiation-induced recombination is dependent on Ku80. Radiat Res 151: 408-413, 1999.
31. Rogakou EP, Boon C, Redon C and Bonner WM: Megabase chromatin domains involved in DNA double strand breaks in vivo. J Cell Biol 146: 905-916, 1999.

32. Rothkamm K, Krüger I, Thompson LH and Lobrich M: Pathways of DNA double-strand break repair during the mammalian cell cycle. Mol Cell Biol 23: 5706-5715, 2003.

33. Camphausen K, Burgan W, Cerra M, Oswald KA, Trepel JB, Lee MJ and Tofilon PJ: Enhanced radiation-induced cell killing and prolongation of $\gamma-\mathrm{H} 2 \mathrm{AX}$ foci expression by the histone deacetylase inhibitor MS-275. Cancer Res 64: 316-321, 2004. 